\title{
French validation of brace questionnaire
}

\author{
Julie Deceuninck*, Jean-Claude Bernard \\ From 11th International Conference on Conservative Management of Spinal Deformities - SOSORT 2014 \\ Annual Meeting \\ Wiesbaden, Germany. 8-10 May 2014
}

\section{Background}

Quality of Life (QoL) Scales have to be introduce in the treatment evaluation of our patients with adolescent idiopathic scoliosis.

Vasiliadis and al. create the Brace Questionnaire $(\mathrm{BrQ})$, the one which is specific for brace treated adolescents. This tool was developed and validated in Greek.

\section{Material and methods}

The BrQ is made of 34 items on Likert Scale, divided in 8 domains. The questionnaire was developed in order that the child could fill in it alone and is adapted for 9 to 18 years old. The lowest scale is 20 and the best 100 . The highest scales show a better QoL.

The process of cultural adaptation of the questionnaire was in accordance with the International Quality of Life Assessment (IQOLA) Guidelines.

\section{Statistical analysis}

Firstly descriptive statistics will be used to calculate mean scores and standard deviations for a given question and a domain. The second level will be comparative concerning reliability and validity. 